\title{
Rethinking Media Synchronicity Theory: Examining the Cooperative Assumption
}

\author{
Jaime Windeler \\ University of Cincinnati \\ windeljb@ucmail.uc.edu
}

\author{
Andrew Harrison \\ University of Cincinnati \\ andrew.harrison@uc.edu
}

\begin{abstract}
Much of information systems (IS) literature assumes team members have completely aligned goals. In practice, people interpret goals to suit personal agendas, even when they are collaborating. This motivates our examination of the cooperative assumption in Media Synchronicity Theory (MST) - a leading IS theory of communication performance. We assess the boundaries of MST by relaxing the assumption of cooperation. Our results support MST for explaining communication and task performance in a cooperative context. However, MST was insufficient to capture how media capabilities influence performance in a non-cooperative context. Our study shows that relaxing the assumption of cooperation changes MST in profound ways-altering which media capabilities are central to the model and the very processes that underlie communication.
\end{abstract}

\section{Introduction}

Cooperation is an underlying assumption in much of the research on computer-mediated communication (CMC) $[1,2,3]$. Indeed, one name for this stream of research is "computer-supported cooperative work" (CSCW). However, even when people come together for a common purpose, agency and opportunism thrive. People conceptualize problems based on their perspective and interpret goals to suit personal agendas. The result is people working together with overlapping, yet incongruent goals that influence how they communicate. This can result in problems for virtual teams that work across functional, geographic, and cultural boundaries. Accordingly, virtual collaborators may have incongruent perspectives and objectives that can compromise task performance.

To explore the implications of non-cooperation for computer-mediated task performance, we ground our study in Media Synchronicity Theory (MST) [4]. MST moves beyond theories of media richness and is geared toward "new media" and information technologies. MST typifies an assumption of cooperation between communication partners. Articulating the boundaries of
MST, Dennis et al. [4, p. 579] say, "We do not specifically address situations where some participants desire to manipulate or control how other participants interact so that the shared understanding that is developed does not reflect the information and opinions of all participants...".

MST is an influential theory in the IS literature; together, the two source papers $[4,5]$ have been cited over 1700 times per Google Scholar and over 500 times per Scopus. Dennis et al. [4] was the MIS Quarterly best paper of the year and the MIS Quarterly Editor-in-Chief named it one of only seven modern "native IS theories" [6]. However, MST has not been empirically tested in its entirety, despite two decades of influence on IS research.

In light of these opportunities to empirically test and expand MST, our research question is: How does MST change when we relax the assumption of cooperation? We approach this investigation from two directions. First, we consider MST in light of noncooperation. This leads us to re-conceptualize constructs in the model and hypothesize additional media capabilities that are relevant when people are not cooperating. Second, we test the model and contrast the results among participants engaged in a cooperative and a non-cooperative communication task. By exploring a prevalent real world context that tends to be ignored - non-cooperation - we add richness to the prevailing view of MST and CMC.

\section{Review of Media Synchronicity Theory}

MST aims to predict communication and task performance given the capabilities of a communication medium. MST posits media possess a set of capabilities that make it suitable for certain communication processes. Whether a set of media capabilities is suited to a task depends on two fundamental communication processes-conveyance and convergence. These processes are supported by capabilities for synchronicity, which refers to working together at the same time with a coordinated pattern of behavior [4]. Lower capabilities for synchronicity are required when information is being conveyed; greater 
capabilities for synchronicity are required when people must converge on meaning $[4,5]$.

In subsequent sections, we review MST starting with the dependent variable in the model and working our way backward to the antecedents. We define key constructs, tenets, and explain how our model extends MST. We then expand MST by identifying media capabilities that are relevant to non-cooperative communication performance.

\subsection{Communication Performance}

Dennis et al. [4] define communication performance, the key dependent variable in their model, as the development of shared understanding. When people are cooperating, shared understanding is a meaningful way to assess communication. However, when people are not cooperating and their personal goals are not aligned, shared understanding may not reflect a successful exchange [7]. In our first departure from MST, we reconceptualize communication performance by distinguishing between cooperative communication performance (for which we use Dennis et al.'s [4] definition) and non-cooperative communication performance.

Cooperation is "the act of working together to one end" [8, p. 8]; non-cooperation represents working together, but relaxes the constraint that a goal is shared amongst communicants [9]. The "ends" to which each person strives may be partially or completely incongruent, and individuals may conceal or distort the information they share with others to garner beneficial outcomes [9, 10]. Non-cooperative communication does not assume that a mutually accepted common goal exists between communicants [9]. This is a departure from MST, which assumes goals are completely congruent.

When people are working toward incongruent goals, they will try to influence one another to maximize their self-interest and achieve their goal [8]. The assertion of social influence through coercion, deception, and persuasion represents one of the most common forms of non-cooperative communication [9, 11]. In this paper we conceptualize non-cooperative communication performance as the extent to which a person can influence others. When a medium enhances one's personal influence over others, there is a greater likelihood of maximizing task performance.

\subsection{Fit, Appropriation, and Processes}

MST proposes that communication performance increases when there is a fit between the communication needs of the task, the capabilities offered by the media, and appropriation factors [4]. This view draws both from the task-technology fit literature [12] and the fit-appropriation model [13] to account for various dimensions of influence on communication performance. Fit is a normative construct capturing a user's perceived match between the needs of the communication process and the capabilities of the medium [4, 14]. Communication processes capture two activities that take place when people communicate: conveying information (i.e., transmission) and converging on meaning (i.e., processing) [15]. Departing from previous research that broadly categorized tasks based on equivocality [30], Dennis et al. [4], had proposed most tasks were rooted in the underlying processes of convergence and conveyance. This more precise conceptualization of task addresses equivocality, task type, and the familiarity or novelty of a task in terms of convergence and conveyance. Thus, MST proposes that all tasks require varying amounts of these processes. Some tasks require a greater focus on converging on shared meaning while other tasks emphasize conveying information. Most tasks require some combination of both processes [4].

Tasks requiring an emphasis on convergence benefit from faster information transmission with more feedback and verification; in contrast, conveyance is a slower, retrospective process [4]. Media provide different capabilities to support information transmission and processing [4]. This yields two key propositions of MST: (1) when convergence on meaning is a goal, media with higher synchronicity will improve communication performance; and (2) when conveyance of information is the goal, media with lower synchronicity will lead to better communication performance.

Finally, appropriation factors are personal and situational characteristics that influence how people use a medium $[4,13]$. MST assumes that appropriation is faithful to the fit of the media and communication process - i.e., that people use the medium as intended for a communication process. We adhere to Dennis et al.'s [4] assumption of faithful appropriation and include their appropriation factors in our model test. These factors are: familiarity with the group, experience with the task and technology, and social norms. In managing our scope, we leave other or unfaithful appropriation factors for future research.

\subsection{Media Capabilities}

Conveyance and convergence rely on messages being passed through media. Media capabilities are "structures provided by a medium which influence the manner in which individuals can transmit and process 
information," [4, p. 583]. These capabilities are physical or socially-derived. Physical media capabilities are objective, observable properties of the media that impact how individuals transmit and process a message [4]. MST includes five physical media capabilities: transmission velocity, parallelism, symbol set variety, rehearsability, and reprocessability.

In contrast, socially-derived capabilities are communication outcomes induced by the physical capabilities of the medium; as such, they may be perceived differently by users depending on the users' experiences and skills [4]. MST includes only one socially-derived capability-media synchronicity-but acknowledge there are others. Socially-derived capabilities have been referred to in extant research as media characteristics [4, 16, 17], capabilities [18, 19, 20, 21], capacities [22, 23], attributes [24, 25], and affordances [26, 27]. For the sake of clarity, we refer to them as socially-derived media capabilities throughout this manuscript.

Due to page restrictions, we exclude the physical media capabilities from the model we present in this paper; we focus instead on the socially-derived capabilities. However, we measured all media capabilities to provide a complete test of MST.

\section{Hypotheses Development}

We hypothesize that media synchronicity will increase perceptions of fit when people are trying to reach a shared understanding (i.e., cooperative task) or assert their personal influence (i.e., non-cooperative task). In other words, we expect a similar effect on fit for both cooperative and non-cooperative communication.

Media synchronicity is "...the extent to which the capabilities of a communication medium enable individuals to achieve synchronicity"-i.e., a shared pattern of coordinated behavior [4]. Media synchronicity enables shared focus among communication partners; media with low synchronicity impedes shared focus [28]. The concept of synchronicity reflects the notion that individuals who share the same understanding can coordinate better. Coordination is important when people are working toward the same goal as it allows them to manage their individual and joint resources efficiently and effectively [29].

Similarly, synchronicity enables people to immediately observe others' actions and responses. This is important when attempting to influence someone's behavior. Delays between sending and receiving a message diminish the emotionality of a message [30], which reduces social norms and thus personal influence. Delays also introduce uncertainty about how a message was received and reduces opportunities to clarify intentions [7]. Lower synchronicity is also associated with filtering out communication cues, particularly body language and facial expression [31]. This may also blunt opportunities to assert personal influence because it reduces the ways a person can communicate their desires.

H1a: Media with greater capabilities for synchronicity will increase perceptions of fit for cooperative communication.

H1b: Media with greater capabilities for synchronicity will increase perceptions of fit for noncooperative communication.

As we considered media capabilities in light of non-cooperation, we reasoned there may be other capabilities besides synchronicity relevant to communication performance. We thus sought to include other media capabilities in the model. Our criteria for relevancy was that the capabilities should support non-cooperative communication performance, i.e., personal influence, and support information transmission and processing. In expanding the media capabilities, it is not our intent to "complete" MST in a systematic way. Our goal is to demonstrate that cooperation is an underlying assumption that influences the media capabilities central to MST.

We reviewed the literature on computer-mediated communication in the IS and communications research domains. We examined papers cited by Dennis et al. $[4,5]$ and papers that have cited MST. From this, we generated a list of media capabilities relevant to communication performance, particularly during noncooperative communication.

Two socially-derived constructs appeared often in the literature that dealt with personal influence: social presence and anonymity [32, 33]. These constructs both capture how the media allows one to perceive others, which is important in controlling perceptions and asserting personal influence. They are not the only capabilities relevant to non-cooperation and they are relevant to cooperation as well. However, they are capabilities IS scholars widely study and are suitable for our research question.

Social presence is the extent to which a medium conveys the physical presence of others (Rice 1992). It is a property of the medium that is perceived rather than observed [34], making it socially-derived. Media that provide more information about communicants is perceived as more "warm, personal, sensitive, and social" [35]. Seeing a person's face or body or hearing their voice makes them seem present, accessible, and real [35]. Research shows social presence increases satisfaction when using web conferencing [36]. 
We expect social presence to increase perceptions of fit when people are trying to reach a shared understanding or assert their personal influence. Research shows that social presence increases the openness of discussion during a cooperative task [37]. It also increases trust and reduces social uncertainty [38]. Research on learning, both in face-to-face and online environments, shows that social presence is important in establishing social attraction and a willingness to work together [36]. This should increase perceptions of fit for communication processes in a cooperative task where shared understanding is the goal. Similarly, social presence should increase perceptions of fit with communication processes for non-cooperative tasks where personal influence is important. Social presence involves an increased awareness of others, creating more social pressure and normative influence $[15,37]$. This makes it easier to assert authority or change others' behavior - behaviors that can be useful in a non-cooperative task.

H2a: Media with greater capabilities for social presence will increase perceptions of fit for cooperative communication.

H2b: Media with greater capabilities for social presence will increase perceptions of fit for noncooperative communication.

Anonymity represents the extent to which a message or information about its sender is perceived to be inaccessible to others [39]. Researchers have consistently found that anonymity influences communication [2, 18, 40, 41]. Anonymity lowers social inhibitions and encourages participation, resulting in a wider range of innovative ideas during group tasks [39]. However, individuals are less likely to consider the interests of others when anonymity is high [42]. Thus, most research exploring the media effects of anonymity has studied whether the desirable effects of increased participation outweigh the potential for antisocial behaviors [13, 40, 42, 43, 44].

We expect different effects of anonymity on fit for cooperative and non-cooperative communication. Anonymity should increase perceptions of fit when people are trying to reach a shared understanding; it should decrease perceptions of fit when people are trying to assert their personal influence. Capabilities for anonymity reduce inhibition and evaluation apprehension, which should be useful for cooperation. Anonymity also increases participation, the number of ideas generated, and improves the quality of decisions [42]. Although social norms and context can temper these effects [45, 46], anonymity is an "equalizer"; it levels the playing field and deflates the influence of any one person. This is important when people share a goal and need to overcome apprehension about contributing or subvert personal agendas.
By this same logic, we expect anonymity will decrease fit in a non-cooperative communication task. Anonymity dehumanizes communication and thus reduces normative pressure [42]. This undercuts personal influence, making it harder to assert desires or to influence others. Anonymity reduces pressure to conform and fear of punishment [2, 18, 40]. Perhaps this is why persuading a stranger on the internet is such a fruitless endeavor.

H3a: Media with greater capabilities for anonymity will increase perceptions of fit for cooperative communication.

H3b: Media with greater capabilities for anonymity will decrease perceptions of fit for non-cooperative communication.

\section{Methodology}

We conducted a laboratory experiment to test our model. Participants are undergraduate students enrolled in introductory courses in a business school at a large Midwestern university in the United States. To date, we have collected data from 736 participants, organized into 184 groups. The experiment has three interaction conditions: face-to-face (F2F), a virtual world using traditional display (VWTD), and a virtual world using a virtual reality display (VWVR).

Virtual worlds (VWs) are computer-based, simulated, persistent environments that support synchronous interaction between users personified as avatars [47]. We chose VWs because they allowed us to compare computer-mediation to face-to-face communication. Face-to-face provides a standard against which scholars often compare technology. Furthermore, they were a good fit for the media capabilities we studied. By using traditional input/output devices (in VWTD) and a head-mounted virtual reality display (in VWVR), we could manipulate social presence. Furthermore, by allowing participants to customize the look of their avatar, display name, and providing one-on-one messaging capabilities, we could manipulate anonymity. Yet, by using very similar technologies, we could hold other media capabilities constant between the conditions.

Both VW conditions employ the Unity platform. The design of the avatars and environments for each condition are identical. The virtual environment models the real world environment as closely as possible. The VW conditions use a game controller for input and headphones with integrated microphone for voice communication. VWTD uses a 22 inch monitor for output. The participant views their avatar in a thirdperson perspective. In contrast, the VWVR condition involves a head-mounted virtual reality display, Oculus Rift SDK2, for output. The participant "sees through 
the eyes" of their avatar in first-person perspective. When they turn their head, the scenes around them change to show other parts of the environment, such as the ceiling or other avatars.

\subsection{Procedure}

After participants registered for the study, they received a link to a survey (T1) that assessed personality and appropriation factors. Upon arrival to the lab, they completed a second survey (T2) that assessed perceptions of media capabilities. After completing the T2 survey, participants were randomly assigned to groups of four and led to a room in preparation for the group task.

In both VW conditions, participants were instructed to arrive at different rooms at different times so that they could not ascertain the identity of their group members. The assignment protocols were designed to ensure that group members had no exposure to the task or each other. Once they arrived at a room, they were seated at a computer and asked to complete the T2 survey. Following this, they accessed the VW platform and customized their avatar's gender, hair, eyes, body shape, and clothing. Next, they performed a training exercise to become familiar with the VW controls.

After the T2 survey and training, all participants performed a cooperative and a non-cooperative group task. The order of the tasks was blocked to control for order effects. Before completing each task, the group watched a video that explained the rules and demonstrated the task. Following this, groups performed the tasks while a researcher recorded data related to the decisions made during the tasks. When both tasks were completed, participants completed a final survey (T3) that assessed perceptions of fit between the tasks and communication media. Participants were then debriefed, paid for their performance, and dismissed. The entire procedure lasted about 1.5 hours per group.

\subsection{Task Description}

We selected two tasks for the experiment. One task was cooperative and required group members to work together, while the other task relaxed assumptions of cooperation. To control for the uncertainty and equivocality of the tasks, the training protocols and task operations are designed to guarantee that each group member is provided the same directions, receives the same level of training, and performs the same actions. The cooperative task is the "Towers of Hanoi" puzzle and has been widely used in social science research [48]. The puzzle consists of three rods (labeled $\operatorname{rod}$ A, B, and C) and four disks of different sizes. The puzzle is placed on a table in the middle of the group. At the start of the puzzle, the disks are stacked in a conical shape on rod A with the smallest disk on top and largest on bottom. The goal of the puzzle is to move the disks to $\operatorname{rod} \mathrm{C}$ in the fewest number of moves, restoring them to their original order. Only one disk can be moved at a time and no disk can be placed on a smaller disk. The minimum number of moves for this task is 16. Participants take turns deciding how to move the disks and discussing their decisions with their group.

For the non-cooperative task, we replicated the "public goods with punishment" game theory design $[49,50]$. This is a variation on a prisoner's dilemma style game that models the difficulty of cooperation between self-interested players. The goal of this game is to maximize individual returns on an investment. The game lasts six rounds. Each round has two phases, an investment phase and a punishment phase. At the start of each round, the investment phase begins and each member of the group is given one dollar in nickels. During the investment phase, all of the subjects simultaneously decide how much money they will contribute towards the group (i.e., the public good) and how much they will keep for themselves. Based on their choices, they are awarded payouts. Any amount invested with the group yields a $40 \%$ return to all players, regardless of whether or not a player invested their own money in the group. Any amount invested individually yields a $10 \%$ return. The payouts are designed to reward groups that act cooperatively; individuals who act selfishly and choose not to invest with the group can reap greater returns.

The punishment phase of the game allows players to coerce others' behavior in subsequent rounds by allowing them to punish selfish group members through monetary penalties. A participant may "pay" nickels to penalize other participants after each round. For each token a participant invests to penalize, the recipient of the penalty will lose 3 nickels. Thus, the penalty is costly for both parties. Sufficiently motivated people will trade the short-term loss associated with penalization for influencing selfish group members to invest with the group. Decisions regarding investments and penalties are made independently and revealed to the group simultaneously. The nickels are placed on game boards with spaces representing investment and penalty decisions. A cardboard box hides the game board from other players until the researcher instructs players to remove the box and reveal their decisions to the group. Winnings are tracked by the researcher and at the end of six rounds, participants receive a payout - the larger of $\$ 10$ or their earnings in the game. 


\subsection{Measurement}

All multi-item measures were adapted from previously validated scales $[2,45,51,52,53,54]$. These measures were assessed on 7-point Likert agreement scales, with the exception of social presence, which was measured using bipolar response. We adapted the measurement items using a multi-step approach [55]. A card sort performed by 92 participants from the same population provided evidence of measurement validity.

For the cooperative task, communication performance was assessed by participants' ratings of how well they understood the group's strategy for completing the Tower of Hanoi puzzle (i.e., shard understanding). For the non-cooperative task, communication performance was assessed by examining whether group members were persuaded to change their investment decisions following punishment (i.e., personal influence).

Following best practices for reducing common method variance, task performance was measured by direct observation. For the cooperative task, task performance was measured by the number of turns taken to solve the puzzle, with lower values representing superior performance. Non-cooperative task performance was assessed by calculating the amount of earnings the groups averaged.

\section{Preliminary Results}

We have gathered data from 736 participants from 184 groups. Of this data, 712 records contain complete information and were used for the analysis. This data allows us to test the general structure of our model and test the validity and reliability of the latent measures. We used components-based structural equation modeling through the SmartPLS 3.0 software package to analyze the data [56]. The 712 records were above recommended thresholds for sample size [21].

Our results indicate our measurement model fits well with a Chi-squared of 4,131.70 and 2,287 degrees of freedom. The normed Chi-squared value is 1.81 , CFI is 0.96 , TLI/NNFI is 0.95 , and RMSEA is 0.03 $[57,58]$. As shown in Table 1 , our data exhibited evidence of reliability, convergent validity, and discriminant validity expected from appropriating prevalidated scales for this study $[59,60]$.

Table 1. Descriptive statistics and correlations

\begin{tabular}{|l|c|c|c|c|c|c|c|c|c|c|c|c|c|c|c|c|c|}
\hline & Mean & SD & AVE & $\mathbf{1}$ & $\mathbf{2}$ & $\mathbf{3}$ & $\mathbf{4}$ & $\mathbf{5}$ & $\mathbf{6}$ & $\mathbf{7}$ & $\mathbf{8}$ & $\mathbf{9}$ & $\mathbf{1 0}$ & $\mathbf{1 1}$ & $\mathbf{1 2}$ & $\mathbf{1 3}$ & $\mathbf{1 4}$ \\
\hline 1. Media Synchronicity & 3.81 & 1.83 & .85 &. $\mathbf{9 2}$ & & & & & & & & & & & & & \\
\hline 2. Social Presence & 4.68 & 1.64 & .62 & .68 & .79 & & & & & & & & & & & & \\
\hline 3. Anonymity & 3.58 & 1.63 & .76 & -.86 & -.60 & .87 & & & & & & & & & & & \\
\hline 4. Communication Process & 5.53 & 1.49 & .85 & -.04 & .05 & .02 & .92 & & & & & & & & & & \\
\hline 5. Experience (Medium) & 2.41 & 1.25 & .66 & .38 & -.35 & .42 & .16 & .81 & & & & & & & & & \\
\hline 6. Experience (Tower Task) & 1.43 & 0.71 & - & -.04 & .05 & -.03 & -.04 & -.11 & - & & & & & & & & \\
\hline 7. Experience (Investment) & 1.61 & 0.83 & - & .05 & -.01 & .01 & -.05 & .00 & .29 & - & & & & & & & \\
\hline 8. Familiarity & 1.43 & 1.46 & - & .11 & -.09 & .04 & -.03 & .03 & .05 & .11 & - & & & & & & \\
\hline 9. Norms & 5.88 & 1.00 & .50 & .02 & -.01 & .08 & .14 & .00 & .06 & -.12 & .05 & .71 & & & & & \\
\hline 10. Fit (C) & 5.87 & 1.08 & .83 & .38 & -.32 & .36 & .32 & .13 & -.01 & -.05 & .00 & .00 & .91 & & & & \\
\hline 11. Fit (N) & 3.52 & 1.54 & .74 & .35 & -.33 & .32 & .04 & .25 & -.04 & .01 & .01 & .21 & .19 & .86 & & & \\
\hline 12. Com Performance (C) & 4.60 & 0.50 & - & .18 & -.17 & .11 & .06 & .14 & .08 & .06 & .04 & .18 & .46 & .07 & - & & \\
\hline 13. Com Performance (N) & 3.64 & 1.35 & .79 & .06 & -.06 & .04 & -.02 & -.03 & -.04 & .02 & -.01 & -.03 & .05 & .45 & .01 & .89 & \\
\hline 14. Task Performance (C) & 19.62 & 5.88 & - & -.11 & .11 & -.10 & .04 & -.07 & -.11 & -.02 & -.09 & -.06 & -.22 & -.04 & -.44 & .01 & - \\
\hline 15. Task Performance (N) & 7.76 & 1.54 & - & -.31 & .30 & -.28 & .00 & -.25 & .03 & .02 & .08 & .05 & -.10 & -.05 & -.08 & .23 & .03 \\
\hline
\end{tabular}

Note: The diagonal shows the square root of the $\mathrm{AVE} ;(\mathrm{C})=$ Cooperative task; $(\mathrm{N})=$ Non-cooperative task; $\mathrm{Com}=$ Communication.

Our tests of MST supported the application of the theory for predicting cooperative behaviors. The results are shown in Figure 1. For a cooperative task, like the Towers of Hanoi, MST was able to predict a substantive amount of variance in communication $(21 \%)$ and task $(41 \%)$ performance.
Consistent with H1a, media synchronicity $(\gamma=.17$, $\mathrm{p}<.001)$ positively influenced fit. Further, communication process $(\gamma=.30, \mathrm{p}<.001)$ had a positive influence on fit indicating that media synchronicity and communication processes increase perceptions of fit for communicating during a cooperative task. While 
appropriation factors did not have consistent effects, our results did support the basic premise that media that fit better for working together at the same time will result improve performance for a cooperative task.

In contrast, MST was insufficient to explain the results related to a non-cooperative task. In the noncooperative task, media synchronicity $(\gamma=.09, \mathrm{p}=.092)$ did not significantly influence perceptions of fit; $\mathrm{H} 1 \mathrm{~b}$ was not supported. Similarly, communication processes $(\gamma=.04, p=.353)$ and appropriation factors also did not significantly influence fit. These findings suggest MST may not be well-suited for explaining non-cooperative communication and that the appropriation factors may not consistently exhibit direct effects on fit.

We also sought to determine if other media capabilities are germane to this model of communication when assumptions of cooperation are relaxed. Our results supported hypotheses $\mathrm{H} 2 \mathrm{a}$ and $\mathrm{H} 2 \mathrm{~b}$, which predicted that social presence would increase perceptions of fit for both cooperative and non-cooperative communication. Social presence increased perceptions of fit in the cooperative $(\gamma=.14$, $\mathrm{p}=.006)$ and non-cooperative $(\gamma=.18, \mathrm{p}<.001)$ contexts.

We also hypothesized anonymity would increase perceptions of fit for cooperative communication in $\mathrm{H} 3 \mathrm{a}$, and decrease perceptions of fit for noncooperative communication in $\mathrm{H} 3 \mathrm{~b}$. Anonymity decreased perceptions of fit in the cooperative $(\gamma=-.14$, $\mathrm{p}=.002)$ and non-cooperative $(\gamma=-.19, \mathrm{p}=.002)$ contexts, supporting $\mathrm{H} 3 \mathrm{a}$ and challenging $\mathrm{H} 3 \mathrm{~b}$.

Consequently, our results suggest that anonymity and social presence could be added to expand the descriptive power of MST-particularly outside of cooperative contexts. Our findings also indicated that anonymity had a negative effect on communication performance during both tasks in the experiment, contradicting research suggesting new teams may benefit from the egalitarian features of anonymity [18]. Instead, our results suggest that anonymity detracted from communication and ultimately performance, even in newly-formed groups.

\section{Future Research and Limitations}

In this study, we identified socially-derived media capabilities that can be used to extend MST to explain non-cooperative communication. However, this list is based on existing theoretical perspectives and does not represent the entire domain of constructs that may affect communication outcomes once assumptions of cooperation are relaxed. We positioned this research to motivate future work to explore other socially-derived media capabilities.

To increase the internal validity of our experiment, our design presented the same task to all respondents. Our emphasis was on understanding how relaxing assumptions of cooperation would affect participants' behaviors. Thus, our research studied conflicting goals, but those goals were not necessarily opposing. Future research may be necessary to determine how a single individual could intentionally undermine group performance.

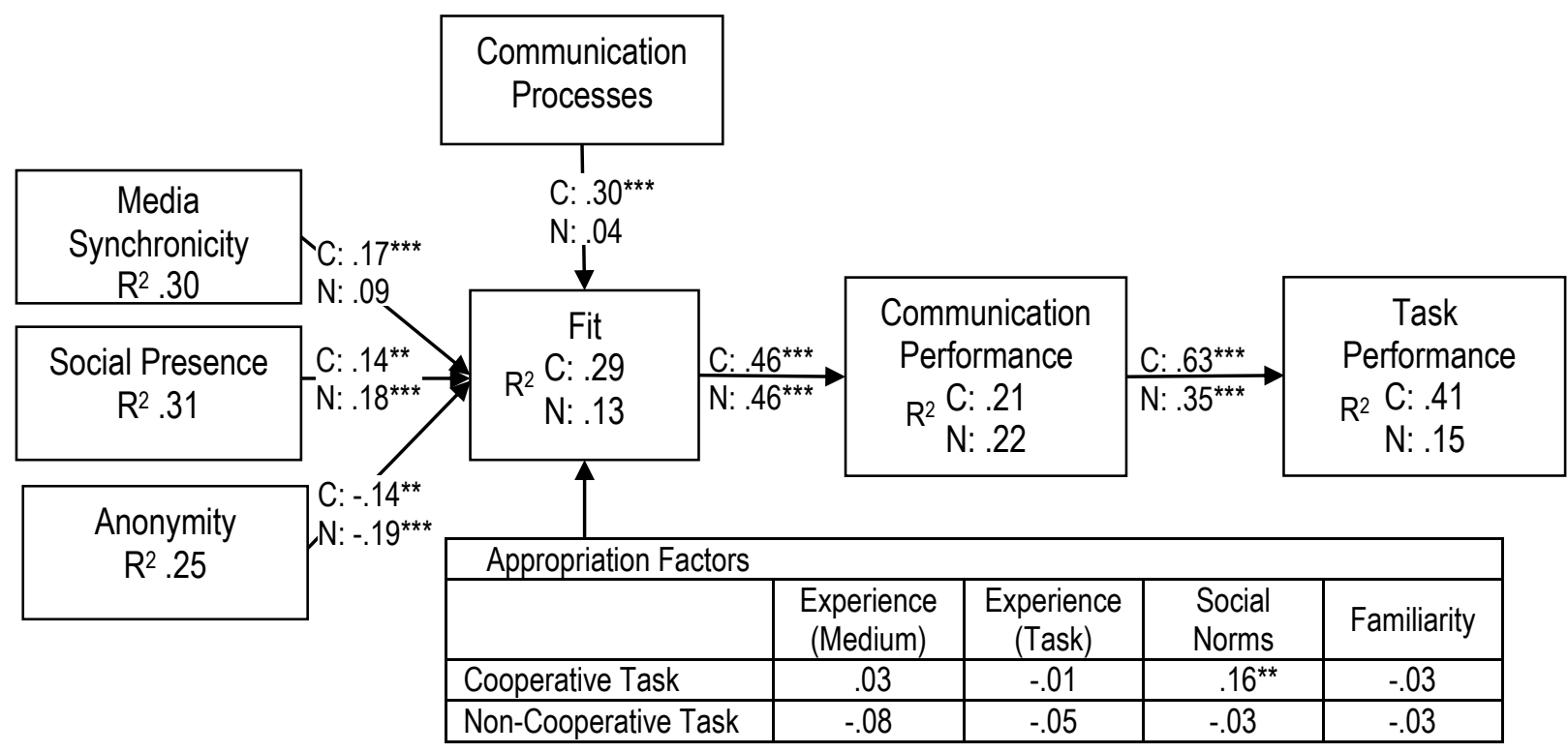

Note: $C=$ Cooperative task; $N=$ Non-cooperative task; ${ }^{*} p<.05,{ }^{* *} p<.01,{ }^{* * *} p<.001$

Figure 1. Analysis results 
This study also provides a foundation for working backwards to unmask the relevant physical media capabilities that act as antecedents to anonymity and social presence. Future research on the relationships between physical and socially-derived media capabilities present an opportunity to examine the design of communication systems that improve communication and task performance [4].

Our study focuses on two modes of communication. During the cooperative task, group members work together towards a shared goal. In the non-cooperative task, group members attempt to influence the decisions of others. However, there are a range of communication modes and intents that extend beyond those explored in this research. Our results suggest future research may use goal congruency to describe how forms of communication are related. A greater understanding of the role of goal congruency provides a basis for integrating context-specific theories like MST [4], Interpersonal Deception Theory [61], and Information Manipulation Theory [10], into general models of communication.

\section{Conclusion}

Based on our preliminary results, we expect to contribute new insights into how media capabilities affect communication processes. In this study, we sought to understand whether the media capabilities that improve communication performance in cooperative exchanges differ from the media capabilities that improve communication in noncooperative contexts. Our study challenges the notion that media synchronicity is the primary means by which media influences communication. Our findings indicate that the inclusion of additional media capabilities are necessary to accurately describe how the capabilities of a medium effect communication performance beyond the bounds of cooperation.

Our study has important implications for expanding research on MST into general domains where a cooperative principle is not assumed [9]. Our findings indicate that social presence may improve communication fit and performance for virtual groups when members pursue different goals. We find the opposite is true of anonymity. Anonymity decreases perceptions of fit, and could detract from communication and task performance when virtual team members' goals do not align. These insights suggest that managers using virtual teams that span across functional, geographic, or cultural boundaries should consider the influence of anonymity and social presence when evaluating prospective information communication technologies.

\section{References}

[1] J.F. George, J.R. Carlson, and J.S. Valacich, "Media Selection as a Strategic Component of Communication", MIS Quarterly, 37(4), 2013, pp. 1233-1251.

[2] S. Sarker, S. Sarker, S. Chatterjee, and J.S. Valacich, "Media Effects on Group Collaboration: An Empirical Examination in an Ethical Decision-Making Context", Decision Sciences, 41(4), 2010, pp. 887-931.

[3] J.S. Valacich, S. Sarker, J. Pratt, and M. Groomer, "Understanding Risk-Taking Behavior of Groups: A "Decision Analysis" Perspective", Decision Support Systems, 46(4), 2009, pp. 902-912.

[4] A.R. Dennis, R.M. Fuller, and J.S. Valacich, "Media, Tasks, and Communication Processes: A Theory of Media Synchronicity", MIS Quarterly, 32(3), 2008, pp. 575-600.

[5] A.R. Dennis, and J.S. Valacich, "Rethinking Media Richness: Towards a Theory of Media Synchronicity", Proceedings of the 32nd Hawaii International Conference on System Sciences, Maui, HI, 1999, pp. 1-10.

[6] D. Straub, "Editorial: Does MIS Have Native Theories?", MIS Quarterly, 36(2), 2012, pp. iii-xii.

[7] J.R. Carlson, and J.F. George, "Media Appropriateness in the Conduct and Discovery of Deceptive Communication: The Relative Influence of Richness and Synchronicity", Group Decision and Negotiation, 13(2), 2004, pp. 191-210.

[8] Mead, M., Cooperation and Competition among Primitive Peoples, Beacon, Boston, MA, 1976.

[9] S.K. Sarangi, and S. Slembrouck, "Non-Cooperation in Communication: A Reassessment of Gricean Pragmatics", Journal of Pragmatics, 17(2), 1992, pp. 117-154.

[10] S.A. Mccornack, "Information Manipulation Theory", Communications Monographs, 59(1), 1992, pp. 1-16.

[11] J. Thomas, "The Dynamics of Discourse: A Pragmatic Analysis of Confrontational Interaction", Unpublished Doctoral Dissertation, Lancaster, England, 1986.

[12] D.L. Goodhue, and R.L. Thompson, "TaskTechnology Fit and Individual Performance", MIS Quarterly, 19(2), 1995, pp. 213-236.

[13] A.R. Dennis, B.H. Wixom, and R.J. Vandenberg, "Understanding Fit and Appropriation Effects in Group Support Systems Via Meta-Analysis", MIS Quarterly, 25(2), 2001, pp. 167-193. 
[14] R.M. Fuller, and A.R. Dennis, "Does Fit Matter? The Impact of Task-Technology Fit and Appropriation on Team Performance in Repeated Tasks", Information Systems Research, 20(1), 2009, pp. 2-17.

[15] S.M. Miranda, and C.S. Saunders, "The Social Construction of Meaning: An Alternative Perspective on Information Sharing", Information Systems Research, 14(1), 2003, pp. 87-106.

[16] B. Choi, and Y. Baek, "Exploring Factors of Media Characteristic Influencing Flow in Learning through Virtual Worlds", Computers \& Education, 57(4), 2011, pp. 2382-2394.

[17] B. Kye, and Y. Kim, "Investigation of the Relationships between Media Characteristics, Presence, Flow, and Learning Effects in Augmented Reality Based Learning", International Journal for Education Media and Technology, 2(1), 2008, pp. 4-14.

[18] T. Carte, and L. Chidambaram, "A Capabilities-Based Theory of Technology Deployment in Diverse Teams: Leapfrogging the Pitfalls of Diversity and Leveraging Its Potential with Collaborative Technology", Journal of the Association for Information Systems, 5(11-12), 2004, pp. 448-471.

[19] R.E. Rice, "Task Analyzability, Use of New Media, and Effectiveness: A Multi-Site Exploration of Media Richness", Organization Science, 3(4), 1992, pp. 475-500.

[20] K. Swan, "Building Learning Communities in Online Courses: The Importance of Interaction", Education, Communication \& Information, 2(1), 2002, pp. 23-49.

[21] L. Yang, and B.C. Tan, "Self-Disclosure on Online Social Networks: Motives, Context Feature, and Media Capabilities", Proceedings of the 33rd International Conference for Information Systems, Orlando, FL, 2012, pp. 1-10.

[22] F. Biocca, C. Harms, and J.K. Burgoon, "Toward a More Robust Theory and Measure of Social Presence: Review and Suggested Criteria", Presence: Teleoperators and Virtual Environments, 12(5), 2003, pp. 456-480.

[23] Y.-T.C. Hung, N.T.T. Duyen, W.-C. Kong, and A.-L. Chua, "Reexamining Media Capacity Theories Using Workplace Instant Messaging", IEEE Transactions on Professional Communication, 51(4), 2008, pp. 352-368.

[24] R.E. Clark, "Media Will Never Influence Learning", Educational Technology Research and Development, 42(2), 1994, pp. 21-29.

[25] L.R. Klein, "Evaluating the Potential of Interactive Media through a New Lens: Search Versus Experience
Goods", Journal of Business Research, 41(3), 1998, pp. 195-203.

[26] D. Halpern, and J. Gibbs, "Social Media as a Catalyst for Online Deliberation? Exploring the Affordances of Facebook and Youtube for Political Expression", Computers in Human Behavior, 29(3), 2013, pp. 11591168 .

[27] K. Kreijns, P.A. Kirschner, and W. Jochems, "The Sociability of Computer-Supported Collaborative Learning Environment", Educational Technology \& Society, 5(1), 2002, pp. 8-22.

[28] S. Münzer, and T. Holmer, "Bridging the Gap between Media Synchronicity and Task Performance: Effects of Media Characteristics on Process Variables and Task Performance Indicators in an Information Pooling Task", Communication Research, 36(1), 2009, pp. 76-103.

[29] M.M. Montoya-Weiss, A.P. Massey, and M. Song, "Getting It Together: Temporal Coordination and Conflict Management in Global Virtual Teams", Academy of Management Journal, 44(6), 2001, pp. 1251-1262.

[30] R.L. Daft, and R.H. Lengel, "Organizational Information Requirements, Media Richness and Structural Design”, Management Science, 32(5), 1986, pp. 554-571.

[31] J.F. George, and J.R. Carlson, "Group Support Systems and Deceptive Communication", Proceedings of the 32nd Hawaii International Conference on System Sciences, Maui, HI, 1999, pp. 1-10.

[32] Blascovich, J., "Social Influence within Immersive Virtual Environments," in The Social Life of Avatars, Schroeder, R. (ed.), Springer, London, 2002, pp. 127-145.

[33] T. Postmes, R. Spears, K. Sakhel, and D. De Groot, "Social Influence in Computer-Mediated Communication: The Effects of Anonymity on Group Behavior", Personality and Social Psychology Bulletin, 27(10), 2001, pp. 12431254.

[34] Short, J., E. Williams, and B. Christie, The Social Psychology of Telecommunications, Wiley \& Sons, 1976.

[35] Y. Yoo, and M. Alavi, "Media and Group Cohesion: Relative Influences on Social Presence, Task Participation, and Group Consensus", MIS Quarterly, 25(3), 2001, pp. 371-390.

[36] C.N. Gunawardena, and F.J. Zittle, "Social Presence as a Predictor of Satisfaction within a Computer-Mediated Conferencing Environment", American Journal of Distance Education, 11(3), 1997, pp. 8-26.

[37] P.B. Lowry, D. Zhang, L. Zhou, and X. Fu, "Effects of Culture, Social Presence, and Group Composition on Trust 
in Technology-Supported Decision-Making Groups", Information Systems Journal, 20(3), 2010, pp. 297-315.

[38] D. Gefen, and D.W. Straub, "Consumer Trust in B2c E-Commerce and the Importance of Social Presence: Experiments in E-Products and E-Services", Omega, 32(6), 2004, pp. 407-424.

[39] T.L. Griffith, and G.B. Northcraft, "Distinguishing between the Forest and the Trees: Media, Features, and Methodology in Electronic Communication Research", Organization Science, 5(2), 1994, pp. 272-285.

[40] T. Connolly, L. Jessup, and J. Valacich, "Effects of Anonymity and Evaluative Tone on Idea Generation in Computer-Mediated Groups", Management Science, 36(6), 1990, pp. 689-703.

[41] S.L. Jarvenpaa, V.S. Rao, and G.P. Huber, "Computer Support for Meetings of Groups Working on Unstructured Problems: A Field Experiment”, MIS Quarterly, 12(4), 1988, pp. 645-666.

[42] J.F. Nunamaker, A.R. Dennis, J.S. Valacich, D. Vogel, and J.F. George, "Electronic Meeting Systems", Communications of the ACM, 34(7), 1991, pp. 40-61.

[43] J.F. Nunamaker, L.M. Applegate, and B.R. Konsynski, "Computer-Aided Deliberation: Model Management and Group Decision Support", Operations Research, 36(6), 1988, pp. 826-848.

[44] J.S. Valacich, A.R. Dennis, and J.F. Nunamaker, "Group Size and Anonymity Effects on ComputerMediated Idea Generation", Small Group Research, 23(1), 1992, pp. 49-73.

[45] A. Pinsonneault, and N. Heppel, "Anonymity in Group Support Systems Research: A New Conceptualization, Measure, and Contingency Framework", Journal of Management Information Systems, 14(3), 1997, pp. 89108.

[46] T. Postmes, and M. Lea, "Social Processes and Group Decision Making: Anonymity in Group Decision Support Systems", Ergonomics, 43(8), 2000, pp. 1252-1274.

[47] C. Parris, "Testimony to the Congressional Hearing on Virtual Worlds", U.S. House Committee on Energy and Commerce, Subcommittee on Telecommunications and the Internet, 2008.

[48] K. Kotovsky, J.R. Hayes, and H.A. Simon, "Why Are Some Problems Hard? Evidence from Tower of Hanoi", Cognitive Psychology, 17(2), 1985, pp. 248-294.

[49] Camerer, C.F., and T.H. Ho. "Behavioral Game Theory, Experiments and Modeling," in Handbook of
Game Theory with Economic Applications, Young, H.P., and Zamir, S. (eds.), Elsevier, Oxford, 2015, pp. 517-573.

[50] E. Fehr, and S. Gächter, "Fairness and Retaliation: The Economics of Reciprocity", The Journal of Economic Perspectives, 14(3), 2000, pp. 159-181.

[51] M. Ahuja, R. Fuller, and M. Magni, "Is Media Synchronicity Theory Culture Blind? An Empirical Test”, Proceedings of the Americas Conference on Information Systems, Puerto Rico, 2015, pp. 1-10.

[52] A.J. Harrison, "The Effects of Technology on Interpersonal Fraud", Unpublished PhD Dissertation, Ames, Iowa, Iowa State University, 2014.

[53] L. Qiu, and I. Benbasat, "An Investigation into the Effects of Text-to-Speech Voice and 3d Avatars on the Perception of Presence and Flow of Live Help in Electronic Commerce", ACM Transactions on Computer-Human Interaction, 12(1), 2005, pp. 329-355.

[54] F. Tang, X. Wang, and C.S. Norman, "An Investigation of the Impact of Media Capabilities and Extraversion on Social Presence and User Satisfaction", Behaviour \& Information Technology, 32(10), 2013, pp. 1060-1073.

[55] Devellis, R.F., Scale Development, Sage Publications, Thousand Oaks, CA, 2012.

[56] C.M. Ringle, S. Wende, and J.M. Becker, SmartPLS 3, Bönningstedt, Germany, SmartPLS, Retrieved from https://www.smartpls.com/, 2015.

[57] Hair Jr, J.F., W.C. Black, B.J. Babin, R.E. Anderson, and R.L. Tatham, Multivariate Data Analysis, PearsonPrentice Hall, Upper Saddle River, NJ, 2006.

[58] L.T. Hu, and P.M. Bentler, "Cutoff Criteria for Fit Indexes in Covariance Structure Analysis: Conventional Criteria Versus New Alternatives", Structural Equation Modeling: A Multidisciplinary Journal, 6(1), 1999, pp. 155.

[59] Chin, W.W., "The Partial Least Squares Approach to Structural Equation Modeling," in Modern Methods for Business Research, Marcoulides, G.A. (ed.), Lawrence Erlbaum Associates, Hillsdale, NJ, 1998, pp. 295-336.

[60] C. Fornell, and D.F. Larcker, "Evaluating Structural Equation Models with Unobservable Variables and Measurement Error", Journal of Marketing Research, 18(1), 1981, pp. 39-50.

[61] D.B. Buller, and J.K. Burgoon, "Interpersonal Deception Theory", Communication Theory, 6(3), 1996, pp. 203-242. 